\title{
Quiet Sun coronal heating: A statistical model
}

\author{
V. Krasnoselskikh ${ }^{1}$, O. Podladchikova ${ }^{1}$, B. Lefebvre ${ }^{2}$, and N. Vilmer ${ }^{3}$ \\ 1 LPCE/CNRS UMR 6115, 3A Av. de la Recherche Scientifique, 45071 Orléans, France \\ 2 ESST, Kyushu University, 6-1 Kasuga-Koen, Fukuoka 816-0943, Japan \\ 3 DASOP, Paris-Meudon Observatory, 5 Pl. J. Janssen, 92195 Meudon, France
}

Received 5 March 2001 / Accepted 16 August 2001

\begin{abstract}
Recent observations of Krucker \& Benz (1998) give strong support to Parker's hypothesis (1988) that small-scale dissipative events make up the main contribution to quiet Sun coronal heating. They also showed that these small-scale events are associated not only with the magnetic network, but also with the cell interiors (Benz \& Krucker 1998). Taking into account in addition the results of the analysis performed by Priest with co-authors (2000) who demonstrated that the heating is quasi-homogeneous along the arcs, we come to the conclusion that the sources driving these dissipative events are also small-scale sources. Typically they are of the order of or smaller than the linear scale of the events observed, that is $<2000 \mathrm{~km}$. To describe statistical properties of quiet Sun corona heating by microflares, nanoflares, and even smaller events, we consider a cellular automata model subject to uniform small-scale driving and dissipation. The model consists of two elements, the magnetic field source supposed to be associated with the small scale hydrodynamic turbulence convected from the photosphere and local dissipation of small scale currents. The dissipation is assumed to be provided by either anomalous resistivity, when the current density exceeds a certain threshold value, or by the magnetic reconnection. The main problem considered is how the statistical characteristics of dissipated energy flow depend upon characteristics of the magnetic field source and on physical mechanism responsible for the magnetic field dissipation. As the threshold value of current is increased, we observe the transition from Gaussian statistics to power-law type. In addition, we find that the dissipation provided by reconnection results in stronger deviations from Gaussian distribution.
\end{abstract}

Key words. Sun: corona - Sun: flares - methods: statistical

\section{Introduction}

Although photospheric convection may supply enough energy to heat the coronal plasma, the way energy is supplied and dissipated in the corona is not completely understood (e.g. Priest et al. 2000; Einaudi \& Velli 1994b). Parker (1988) suggested that the solar corona could be heated by the dissipation at many small-scale tangential discontinuities arising spontaneously in the coronal magnetic fields braided and twisted by random photospheric footpoint motions. To emphasize these events, he introduced the notion of nanoflares. This idea stimulated the intensive search of observational signatures of microflares and nanoflares as well as many theoretical developments on the contribution of small scales to energy dissipation in the solar corona. The solar flares, the most energetic impulsive phenomena in active regions, were observed and studied in detail for quite a long time. On a large scale, it is well known that the most energetic dissipative events

Send offprint requests to: V. Krasnoselskikh, e-mail: vkrasnos@cnrs-orleans.fr occur in the vicinity of tangential discontinuities at the borders of the photospheric dipolar regions like bright spots. These sources create strong currents resulting in reconnection and flare-like events. Such eruptive events are related to magnetic energy releases, sudden changes of the magnetic field topology, and plasma heating. Therefore Parker's idea about solar corona heating by nanoflares used quite similar physical concepts about the nature of dissipative phenomena, but it pointed out the importance of small scales.

The smaller-scale phenomena, microflares, were first detected in soft X-rays in a balloon experiment by Lin et al. (1984). The development of new instrumentation allowed performing the multi-wave satellite (YOKOH, SOHO, TRACE) and ground based (VLA) high-resolution observations of smaller-scale (about a thousand of kilometer) lower energy phenomena. They were observed in active regions but also in the quiet regions of the Sun and in coronal holes. The small intensity flares observed in active regions were quite rare and not powerful enough for heating (Shimizu et al. 1994). These observations were 
performed in the regions of high levels of background flux and strong stray light. The very same microflares can be more easily detected in the quiet corona. Soft X-ray observations (Benz et al. 1997) and EUV observations (Harrison 1997) have revealed enhanced emission and thus intense heating above the magnetic network. A similar phenomenon that forms small X-ray jets at the limb was reported by Koutchmy et al. (1997). It was found that the number of observed events increases with the sensitivity.

Another way to shed some light on the important problem concerning the characteristic scales of the major dissipative events consists of statistical study of different observed parameters. The major objective of such a study is to obtain the energy Probability Density Function (PDF) over a wide range of energies.

For regular flares that occur mainly in active regions, Crosby et al. (1993) have found that the total energy in the flare electrons observed in hard X-ray bremsstrahlung has a power law distribution $f(E) \sim E^{\alpha}$ with index $\alpha=$ $-1.53 \pm 0.02$. But the energy supplied by the flares in the active regions is not sufficient for the corona heating.

The crucial factor that determines the characteristics of the heating process and its scales is related to the power law index of the PDF. If it is larger than minus two the major heating takes place in the intensive large-scale dissipative events. If it less than minus two it occurs in small scales. The reason is that to provide the explanation of the efficient coronal heating at small scales, it is necessary to have an excess in the occurrence rate of small-scale events. An important result that supports the hypothesis of Parker was reported by Krucker \& Benz (1998) who have found from the Yohkoh/SXT observations that the PDF dependence upon the energy has a power law distribution in the energy range $10^{24}-10^{26}$ ergs with the index about -2.59 . This result was obtained assuming that the flaring region has a constant height. The authors not only concluded that the weak flaring events rule the heating process, but they also noticed that the brightest small scale microflares lie above enhanced elements of the magnetic network, and the denser ones exhibit a higher level of fluctuations. This means that the corona is continuously replenished by underlying chromospheric material that has been heated to coronal temperatures. Mitra \& Benz (2000) have discussed the same observations but supposing that the height varies proportionally to the square root of the area and have shown that the index becomes a little larger but still is smaller than minus two. This result was confirmed by Parnell \& Jupp (2000), who estimated the index to be between -2 and -2.1 making use of the data of TRACE. However, Aschwanden et al. (2000), also using the data of TRACE, have found significantly different value of this index -1.80 , suggesting that there may not be enough energy in microflares or nanoflares to heat the entire quiet corona. These last authors have cast doubt on the hypothesis that the heating takes place at small scales, which according to them remains controversial.

It is also worth mentioning that Benz \& Krucker (1998) and Berghmans et al. (1998) have noticed that the heating events occur not only on the magnetic network boundaries but also in the cell interiors. They came to the conclusion that these events have a quite similar nature.

The idea of small-scale intermittent behavior of dissipation was elaborated in theoretical models by several authors. Using an MHD approach, Einaudi \& Velli (1994a) investigated the dependence of the dissipated energy upon the scales. Different kinds of "fragmentation of dissipation" were discussed by Vlahos (1994). Einaudi et al. (1996) and Georgoulis et al. (1998) simulated 2D MHD systems driven by large-scale forces and with diffusive dissipation. Their 2D simulations, driven by a pair of largescale vortices with random phases and amplitudes, showed that the spatial average of the dissipated power had nonGaussian statistics. After substraction of the Gaussian component, the dissipated events were shown to have a scaling law distribution.

The observed power-law distributions of the energy flux for the largest dissipative events, flares, and microflares inspired the development of models based on the idea of Self-Organized Criticality (SOC) (Lu \& Hamilton 1991; Lu et al. 1993; Vlahos et al. 1995; Georgoulis et al. 1995; Georgoulis \& Vlahos 1996, 1998). Lu et al. (1993) and Georgoulis et al. (2001) compared the predictions of the models with the observations of the flares. SOC has appeared as a paradigm for slowly driven complex systems, which exhibit power-law-type relaxation events and correlations of infinite range (Jensen 1998). These models are similar to the original "sandpile" model (Bak et al. 1988), but the sand is replaced by the magnetic field flux. The dissipation occurs through small-scale reconnection, when a "current", which is defined in terms of the difference between the field in a particular cell and the average over all nearest neighbors, exceeds some threshold. In these models, a perturbation in a single cell can trigger quite a large cascade of reconnections. This results in a power-law energy distribution of dissipative events and power-law spatial correlations. However, one should take care in interpreting these results because a small grid size can result in an artificial form of the PDF's obtained. A heuristic justification of such models based on 2D MHD equations was proposed by Vassiliadis et al. (1998). It is also worth mentioning that, using a shell model of MHD turbulence, Boffetta et al. (1999) have demonstrated that there can exist alternative reasons for a scaling law to appear.

Our model is also based on the idea of cellular automata. Its difference with the previous ones consists in the assumption that not only the dissipation takes place in small scales but the magnetic field sources are also of small scale. This is in agreement with the idea of Benz \& Krucker (1998) that the heating can take place on the level of the chromosphere, implying that the magnetic field structures have the scales of the order of or less than its height. This feature results from the analysis of Priest with co-authors (1998) who have shown that the heating is quasi-homogeneous over the height of the magnetic loop. Other indications about the possible role of the 
small-scale sources follow from observations of Abramenko et al. (1999) of the evolution of the vertical component of the current helicity of an active region magnetic field during a transition from a low flaring state to an enhanced one. They found that the reorganization of the vortex structure of the photospheric magnetic field occurs, when small scale vortices re-organize into larger scale vortices, suggesting that an inverse helicity cascade develops.

Although the footpoints of the loops are known to move randomly, the statistical properties of their motions are not known in detail. These sources create strong currents resulting in reconnection and flare-like events. Such eruptive events are related to magnetic energy releases, sudden changes of the magnetic field topology, acceleration of particles and plasma heating. Another possible source of energy dissipation and coronal heating is provided by an anomalous resistivity resulting from currentdriven instabilities which are developed when the currents exceed a certain threshold value. It provides Joule-like dissipation in collisionless plasma. This results in a relatively slow heating, but does not give rise to particle acceleration as for the previous dissipation mechanism. Moreover it may occur everywhere in the turbulent shear flows and not necessarily on the boundaries of the magnetic field network.

Another important feature that makes the difference from previous studies of CA type models is the homogeneous distribution of the magnetic field sources. The problem to be addressed in the present work is whether the small-scale magnetic fields generated in (or convected from) the photosphere or chromosphere (mainly at the boundaries between granulas, but also inside them) can make a significant contribution to the coronal heating.

We are mostly interested in the scales typical for nanoflares and even in smaller ones. In this case, the smallscale sources of the magnetic field and the energy dissipation can have comparable characteristic spatial scales. Thus the inertial interval as well as the energy cascade are not as important in such a model as for the conventional Alfvénic or MHD turbulence. But the system considered can possess the property of "inverse cascade", thereby influencing the structure of the larger-scale magnetic fields, as is supposed to be the case in the SOC-type systems. In this paper we concentrate on the study of the dissipated energy flow, in particular, on the dependence of statistical characteristics of dissipated energy flow upon the characteristics of the magnetic field source, and on the physical mechanism of the magnetic field dissipation.

\section{Small scale driving and dissipation}

We consider hereafter the sand-pile-type cellular automata, where sand piles are replaced by a magnetic field flux. In the model, the relaxation process consists of the dissipation of the magnetic field energy by means of the dissipation of currents, when their magnitudes exceed a certain threshold value, or due to the reconnection process.

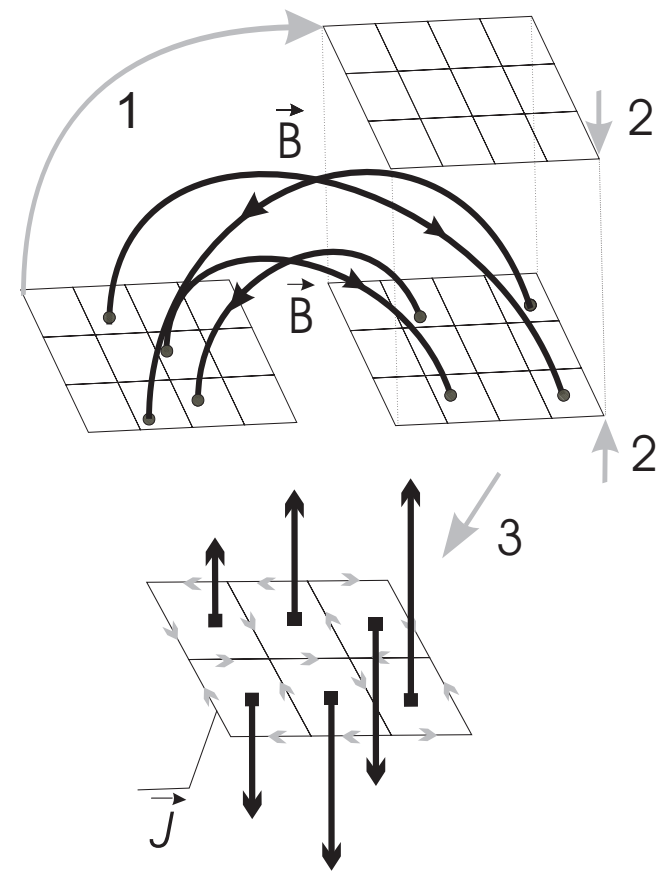

Fig. 1. A schematic representation of the procedure used in our model to simplify magnetic field configuration.

In MHD approximation the evolution of the magnetic field is governed by

$\frac{\partial \boldsymbol{B}}{\partial t}=\nabla \times(\boldsymbol{u} \times \boldsymbol{B})+$ dissipative term,

where $\boldsymbol{B}$ is the magnetic field, $\boldsymbol{u}$ is the fluid velocity of the plasma. Without the dissipative term, this equation describes the motion of the magnetic field lines frozen into the plasma. In this case, the reconnection is forbidden. When a finite conductivity $\sigma$ is taken into account in the form of Ohm's law $\boldsymbol{j}=\sigma(\boldsymbol{E}+\boldsymbol{u} \times \boldsymbol{B})$, where $\boldsymbol{j}$ is the current density, it gives rise to the diffusion effect. In Eq. (1) the effect is described by a dissipative term $\eta \Delta \boldsymbol{B}$, where $\eta=1 / \sigma \mu_{0}$ and $\mu_{0}$ is the magnetic permeability of the free space. The diffusion time $t_{\eta}=l^{2} / \eta$ is generally large for all scales but small where it is comparable with the typical time for fast reconnection. In this case it has the same order of magnitude as an Alfvén time $t_{\mathrm{A}}=l / V_{\mathrm{A}}$, where $V_{\mathrm{A}}$ is the Alfvén velocity. It is worth mentioning that at small scales other dissipative effects can become important (see, e.g., Biskamp 1993).

In the present study, we use a simplified cellular automata model mimicking Eq. (1).

Assume that the curvature of the magnetic field lines is negligible. Then, in each cubic column only the upper and lower faces should be considered, i.e., magnetic field lines are straight and perpendicular to the surface. In this case the equation $\nabla \cdot \boldsymbol{B}=0$ is automatically satisfied (see Fig. 1). As a result, the fields on the lower and upper faces are equal, thus only one face will be considered. The $2 \mathrm{D}$ surface is then split into cells. In most of the simulations presented in the paper, grids of $200 \times 200$ cells are used 
(unless otherwise stated). The boundary conditions are chosen to be periodic.

As we have mentioned already in the introduction Krucker \& Benz (1998) have found that the main heating occurs in the small scale bright points. Making use of the multi-wavelength analysis Benz \& Krucker (1999) have shown that the temporal sequence of observations of different wave emissions is similar to the one in the large scale magnetic loops. They came to the conclusion that the physical mechanisms of the energy release are similar in these two cases. It follows then that the dissipation process in the second case is also associated with the magnetic loops, but at a small scale. Moreover, they have noticed that the heating events occur not only on the boundaries of the magnetic network but in the interiors of the cells also. The comparative analysis of the model predictions for the plasma heating in the magnetic loop due to the distributed energy source with observations performed by Priest with co-authors (1998) led to the conclusion that the heating is quasi-homogeneous along the magnetic loop. This means that the heating process does not occur in the close vicinity of the foot points but rather in the whole arc volume. If one will put together these facts, it follows that the characteristic spatial scale of the magnetic field loops which supply the magnetic field dissipated is of the same order as the characteristic scale of the dissipation. Thus we may conclude that not only the dissipative process, but also the sources, have small characteristic length. Another conclusion from observations is that the sources are distributed quite homogeneously in space. This reasoning leads us to the choice of the sources and dissipation mechanisms used in our model that we describe further.

Three kinds of sources with slightly different statistics are considered.

\subsection{Source terms}

We investigate the statistical behavior of the system driven by a random and turbulent unipolar/dipolar sources. These sources are used to model the effects of turbulent magnetic field convection described by the first term on the right-hand-side in Eq. (1).

- An unipolar random source. The simplest source of magnetic energy is an uncorrelated process of zero mean, $\langle\delta B\rangle=0$, the values of which are randomly chosen from the set $\{-1,0,1\}$, all the values being equiprobable. In each time step the action of the source consists of adding random numbers from the set mentioned above to the previous values in the cell. The numbers are independently chosen for each cell. This procedure automatically ensures that $\langle\delta B\rangle=0$ for each cell.

- A dipolar random source. Such a source can be made dipolar by dividing the grid into two parts. For the positive and negative parts of the grid the random numbers $\langle\delta B\rangle=0$ are chosen from the sets $\{-0.5,0.5,1.5\}$ and $\{-1.5,-0.5,0.5\}$, respectively.
- A chaotic source. Turbulence is certainly not a completely random process, and some of its aspects can be simulated using deterministic models. The Ulam map provides one of the simplest examples of a generic chaotic system with quadratic non-linearity (see, e.g., Frisch 1995). The source $\delta B$ in each cell evolves according to

$\delta B_{n+1}=1-2\left(\delta B_{n}\right)^{2}$,

where the initial values of $\delta B_{0}$ are randomly chosen from the interval $[-1,1]$. In this case, all other $\delta B_{n}$ belong to the same interval. The action of the source is similar to the above described for random sources.

- A Geisel map source. In physical systems, the source term may depend on the value of $B$ itself. When the dissipation is absent, the magnetic field in each cell evolves according to the map

$B_{n+1}=f\left(B_{n}\right)$.

The initial values of $B_{0}$ are randomly chosen from the interval $[-0.5,0.5]$. The systems like that are usually called Coupled Map Lattices (CML) (Kaneko 1992) rather than cellular automata. We use the source based on the Geisel map (Geisel \& Thomae 1984) shown in Fig. 2. The fixed points of this map that are defined by $B_{n}=f\left(B_{n}\right)$ are metastable. As a result, an intermittency develops in the system, i.e., the dynamics is slow and regular in the vicinity of the fixed points of the map and fast and chaotic otherwise. As a consequence, the map that describes the time evolution of the magnetic field in each particular cell exhibits behavior similar to anomalous (non-Brownian) diffusion, i.e.

$$
\left\langle B^{2}\right\rangle \propto t^{\alpha}, \quad \alpha<1
$$

The hypothesis has been suggested that when the turbulence consists of convective cells, magnetic field lines time evolution in each cell is similar to a subdiffusive behavior $(\alpha<1)$, which is, however, more complex than described above.

\subsection{Dissipation criteria}

The magnetic field dissipation provides the conversion of the magnetic energy into thermal energy and ensures the coupling between the magnetic field elements in our model. Phenomenologically, reconnection can be treated as a dissipation of small-scale current sheets when the current density exceeds a certain threshold value (Somov \& Syrovatsky 1977; Syrovatsky 1981, 1982).

If we neglect the displacement current, the current density can be calculated from Maxwell-Ampère's law $\nabla \times \boldsymbol{B}=\mu_{0} \boldsymbol{j}$, the finite-difference form of which can be written as

$\left(\begin{array}{l}j_{x} \\ j_{y}\end{array}\right)=\frac{1}{\delta \mu_{0}}\left(\begin{array}{c}B(x, y)-B(x, y+\delta) \\ B(x+\delta, y)-B(x, y)\end{array}\right)$, 


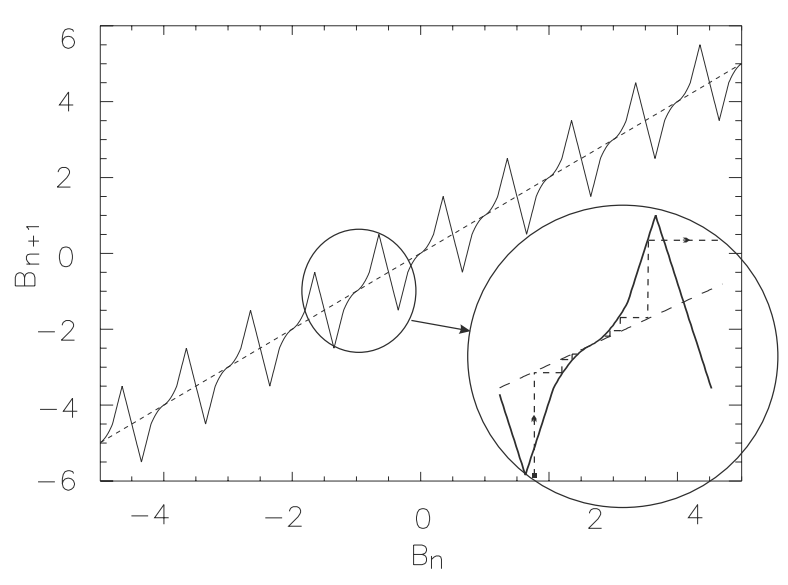

Fig. 2. Graphical representation of the Geisel map (solid line). The fixed points of the map correspond to the intersections of the graph with the straight line $B_{n+1}=B_{n}$ (dashed line).

where $\delta$ is the grid increment. For simplicity we let $\delta=1$ and $\mu_{0}=1$. Currents are thus computed as local gradients, and supposed to be carried along the borders between the cells. It is seen that the discrete analog of the current continuity equation $\nabla \cdot \boldsymbol{j}=0$ holds, i.e., the sum of incoming and outgoing currents is equal to zero at each node of the grid.

The mechanisms for the current dissipation are of two types.

- Anomalous resistivity arises as the result of an instability when the electric current exceeds some critical value (which may be sometimes very small), and results in an increase of the plasma's resistivity. This phenomenon describes the phenomenon of pulse and energy exchange between electrons and ions, or between different groups of particles of the same nature by means of plasma turbulence in collisionless plasma (Galeev \& Sagdeev 1979, 1984).

When the electric current in plasma exceeds a certain threshold value, plasma instabilities can be excited. As a result of the instability development, the waves are generated (e.g. drift waves, or ion-acoustic, or low-hybrid waves). The development of the instability gives rise to the absorption by the generated waves of a part of the electron's energy and pulse which is partly redistributed to ions. This results in a dissipation of the current quite similar to Joule heating (see Appendix A for more details). Finally, it is worth noting that anomalous resistivity does not require any particular configuration of the magnetic field, besides the strong current, and may occur even in the presence of parallel magnetic fields pointing in the same direction, as for example in coronal holes or cell interiors. In our model we assume that the currents are completely annihilated whenever they exceed a certain threshold,

$|j| \geq j_{\max }$

- Reconnection is generally understood as a relatively sudden change from one equilibrium state to another, implying a change in the magnetic field's topology, accompanied by a transformation of magnetic energy into energy of particules (Priest \& Forbes 2000). In its "primary form" the stationary reconnection process represents the dissipation of the magnetic field in the vicinity of the so-called $X$-points of the magnetic field configuration or in the vicinity of a current sheet. That can be interpreted as the rising and "burnout" of thin small-scale current sheets separating domains with oppositely directed magnetic fields. To mimic this process, we assume that the following two conditions should be satisfied simultaneously,

$$
\begin{aligned}
& |j|=\left|B-B^{\prime}\right| \geq j_{\max }, \\
& B \cdot B^{\prime}<0,
\end{aligned}
$$

where $B$ and $B^{\prime}$ denote the magnitudes of the magnetic field in neighboring cells. These conditions are supposed to hold for conventional plane reconnection configuration with an $X$-point (Petchek 1964; Syrovatsky 1981). Equation (2) results in the existence of currents that can significantly exceed the critical value (more details can be found in Appendix B).

This is taken into account by requiring that magnetic fields in adjacent cells have opposite directions, which supposes the existence of a magnetic null point in between, and corresponds to an unstable equilibrium favorable to reconnection.

This seems to be enough to distinguish between the two dissipation mechanisms in the framework of the cellular automata model, imposing that the reconnection requires a special configuration (an $X$-point for instance), whereas the only criteria for anomalous resistivity is a current greater than a given threshold. Indeed, magnetic reconnections corresponds to an important change in the magnetic field's topology, whereas anomalous resistivity does not. In real physical conditions of the corona, both processes can be present. The threshold current for the anomalous resistivity is in general supposed to be much larger than for the reconnection under similar plasma parameters. However our task in this work is confined to the study of the statistical properties of the energy dissipation dependence upon parameters. Closer comparison with real parameters in the corona will be the object of a more detailed study in the future and is beyond the scope of this paper.

The rules that we use to describe the dissipation process are based on the magnetic field dissipation, that means for us transformation to particle energy or heating. For the sake of simplicity and pure formulation of the problem we consider here that all the magnetic field energy is transformed completely into heating. The rules are as follows: when the current is annihilated, magnetic field values in both neighboring cells, $B$ and $B^{\prime}$, are replaced by $1 / 2\left(B+B^{\prime}\right)$, thus, the density of magnetic energy dissipated in a single event is given by

$\Delta E=\frac{1}{2}\left(B-B^{\prime}\right)^{2}=\frac{1}{2} j^{2} \gtrsim \frac{1}{2} j_{\max }^{2}$. 
The procedure modeling the dissipation of currents is the same for both anomalous resistivity and reconnection. For each time step, the currents satisfying the dissipation criterion are dissipated until all the currents become subcritical (or have the same sign in the case of reconnection). Then, we proceed to the next time step and switch on the source. Indeed, dissipative processes are supposed to be faster than the driving ones. The total dissipated energy is calculated as a sum over all the dissipated currents for the time step considered.

To compare the effect of a single act of the magnetic field driver with that of the energy dissipation, one can analyze the spatial Fourier transform of the energy dissipation and the energy influx,

$$
\begin{aligned}
& I_{\text {source }}(k)=\frac{16}{k_{x}^{2} k_{y}^{2}} \sin ^{2}\left(\frac{k_{x}}{2}\right) \sin ^{2}\left(\frac{k_{y}}{2}\right), \\
& I_{\text {diss }}(k)=\frac{16 j_{\max }^{2}}{k_{x}^{2} k_{y}^{2}} \sin ^{4}\left(\frac{k_{x}}{2}\right) \sin ^{2}\left(\frac{k_{y}}{2}\right),
\end{aligned}
$$

where $k_{x}, k_{y} \in[-\pi, \pi]$. The last equation holds for the dissipation of the $y$-component of current. For $x$-component $k_{x}$ and $k_{y}$ should be interchanged.

It is seen that the dependence of these two spectra on $k_{x}$ are quite different. For each fixed $k_{y}$, the energy dissipation vanishes at $k_{x}=0$ and increases monotonously with the growth of $\left|k_{x}\right|$, while the source of the magnetic field is maximum at $k_{x}=0$ and decreases with the increase in $\left|k_{x}\right|$. It is worth noting that for large thresholds, one act of dissipation takes place after a large number of actions of the magnetic field influx, having different phases on each step, which are randomly distributed. This difference in the action of the magnetic field source versus dissipation introduces some intermediate scale in the $k$-space where the action of the source is approximately compensated by the action of energy dissipation. This characteristic value of $k$ is closely related to the characteristic correlation length of the magnetic field spatial distribution. The growth rate of the magnetic field energy and the effective damping rate are non-vanishing almost everywhere in the $k$-space, but the growth rate dominates for small $k$ 's (large spatial scales) while dissipation dominates for larger $k$ 's (smaller spatial scales). Such a situation corresponds in terms of the energy cascade to the "normal" direction of the energy flux, i.e., from large scales to smaller ones. It is also worth mentioning that using uniform driving results in an important difference between our model and conventional SOC models, where the extreme tenuousness of the driving is essential (Sornette et al. 1995). This tenuousness makes the driver nonlocal, in the sense that it depends on the state of the whole system, as discussed by Vespigniani \& Zapperi (1998).

\subsection{Characteristic spatial scales}

Dissipation mechanisms and their thresholds depend upon the parameters of the plasma of solar corona such as background magnetic field, density, etc. Since our model is aimed at describing local regions in the corona rather than the corona as a whole, it is quite natural to assume that the same dissipation criterion can be applied for each cell of the grid. Thus the question about the characteristic sizes of the dissipated currents arises. The observations and theoretical studies show that the scale of current sheets can be smaller than $1 \mathrm{~km}$. The smallest scales, of about $10 \mathrm{~m}$, which are considered by Einaudi \& Velli (1999), are associated with the current regions for the Petchek-type reconnection events. Let us estimate the characteristic scales of the dissipation events due to anomalous resistivity. Assuming that the resistivity is provided by the ion sound instability that has quite a small threshold, we can easily obtain

$|\nabla \times \boldsymbol{B}| \simeq B / L>\frac{4 \pi}{c} n_{\mathrm{e}} e c_{\mathrm{s}}$,

where $L$ is a characteristic width of the current sheet layer, $c$ is the speed of light, $B$ is the characteristic magnitude of the magnetic field, $n_{\mathrm{e}}$ is the plasma density, and $c_{\mathrm{s}}=$ $\left(T_{\mathrm{e}} / m_{\mathrm{i}}\right)^{1 / 2}=\left(m_{\mathrm{e}} / m_{\mathrm{i}}\right)^{1 / 2} v_{T \mathrm{e}}$ is the ion-sound velocity, $T_{\mathrm{e}}$ is the electron temperature, $v_{T \mathrm{e}}$ is the electron thermal velocity, $m_{\mathrm{e}, \mathrm{i}}$ are the electron and ion masses. Then

$L<\frac{B c}{4 \pi n e v_{T \mathrm{e}}}\left(\frac{m_{\mathrm{i}}}{m_{\mathrm{e}}}\right)^{1 / 2}=\beta^{-1 / 2} \frac{c}{\omega_{\mathrm{pi}}}$,

where $\omega_{\mathrm{pi}}$ is ion plasma frequency and $\beta$ is the ratio of the kinetic to magnetic pressure. In the low corona, where $\beta$ is supposed to be of the order of 1 , we have $L \simeq 300 \mathrm{~m}$. This scale is significantly smaller than the spatial resolution of modern experimental devices. Moreover, using the angular scattering measurements of the electron density fluctuations, the smallest scale that can be resolved in the slow solar wind at $8 R_{\odot}$ is $6 \mathrm{~km}$ (Woo \& Habbal 1997). Assuming that the linear structures expand radially as $r$, the structures at $1 R_{\odot}$, where dissipation is supposed to occur, are of the order of $1 \mathrm{~km}$.

Thus, until now, only macroscopic characteristics can be observed. The statistical microscopic models are aimed at reproducing the main features of these observations. In this paper we study the influence of the statistical properties of the magnetic field source, of the type of dissipation mechanisms and their thresholds on the macroscopic properties of the total flux of dissipated energy. This problem is related to the other ones. In particular, can the local mechanisms of dissipation result in long-range spatial correlations? In self-organized systems, the appearance of such correlations give rise to power law distributions of dissipated energy. Such properties may be caused by specific features of the source such as the deterministic chaos or non-Brownian diffusion.

\section{Results}

Preliminary results concerned with the influence of the type of magnetic field energy dissipation on statistical properties of the total radiation energy flux were presented 


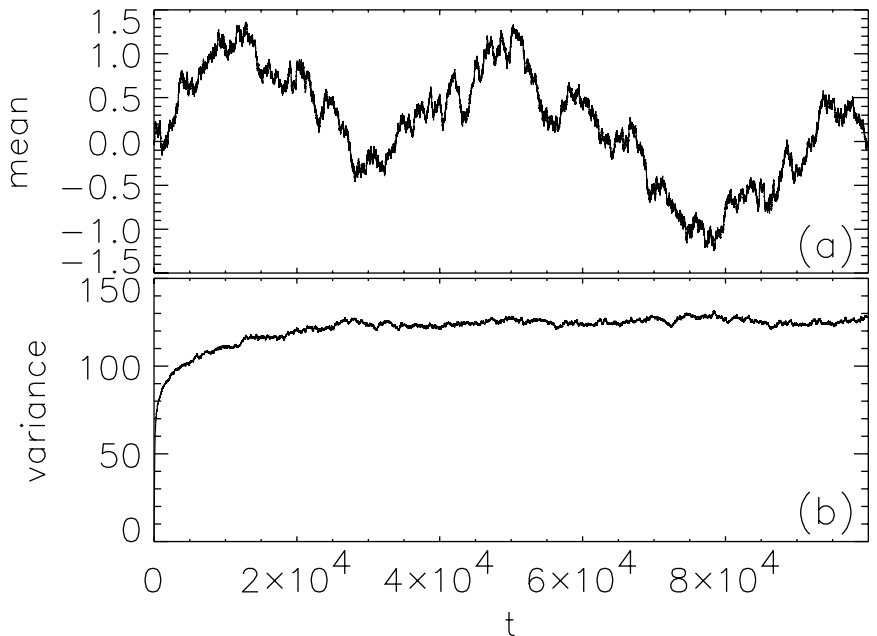

Fig. 3. Evolution of mean and variance of the magnetic field. For variance, observed are three stages - a linear increase, a nonlinear stage, and a stationary state. The results are obtained for unipolar random source and anomalous resistivity dissipation with a threshold $j_{\max }=30$.

by Podladchikova et al. (1999). It was shown that the dissipated energy has approximately normal distribution when the source of the magnetic field is random and the dissipation is provided by anomalous resistivity. This result was obtained under the condition that the current density threshold for the dissipation to occur was moderate $\left(j_{\max }=5\right)$. Under the very same conditions, but for the dissipation due to reconnection, the non-Gaussian energy tails were observed as well as some other interesting features, in particular, large-scale spatial correlations of the magnetic field. This result seems to be quite natural because in this case some currents may exceed the critical value but not dissipate when the magnetic fields have the same direction in the neighboring cells (see Eq. (2)). The currents may grow to larger magnitudes and provide more intensive energy releases in a single dissipative event and sometimes longer chains of these events. The large energy events are those that result in significant deviations from the Gaussian distribution.

\subsection{Transient and stationary states}

We have studied the dependence of the statistical properties of our system upon the dissipation threshold $j_{\max }$ ranging from 0.01 to 300 . To perform a statistical analysis correctly, the averaging procedures should be carried out for the stationary state of the system. The transient time depends on the threshold.

If the dissipation is absent, the field in each particular cell would follow a Brownian motion under the influence of the random source. As a result, the magnetic field in each particular cell, as well as the average over the whole box, would have a Gaussian distribution with a growing variance, $\left\langle B^{2}\right\rangle \propto t$. The currents exhibit the similar behavior, the current variance growing as

$\left\langle j^{2}\right\rangle(t) \approx 2\left\langle\delta B^{2}\right\rangle t$

where for the random source $\left\langle\delta B^{2}\right\rangle=2 / 3$. On the average, the current dissipates for the first time at time

$t_{\mathrm{S}} \simeq \frac{j_{\max }^{2}}{2\left\langle\delta B^{2}\right\rangle}$

This relationship also gives a characteristic time between two dissipation events that occur in the same cell in a stationary state. To obtain reliable statistics, the simulation time should significantly exceed $t_{\mathrm{S}}$. Depending on $j_{\max }$, the simulations performed have $10^{5}-10^{6}$ time steps.

Dissipation finally saturates the growth of the variance (see Fig. 3b), and a stationary state is reached. The average magnetic field $B$ over the whole grid undergoes strong fluctuations but its time average is zero, Fig. 3a). The average number $n$ of dissipated currents at each time step can be estimated from the energy balance considerations. For a single time step, the energy input on a $N \times N$ grid is

$\delta E_{\text {in }} \simeq N^{2}\left\langle\delta B^{2}\right\rangle$,

while the dissipated energy is

$\delta E_{\mathrm{diss}} \simeq-n \frac{j_{\max }^{2}}{4}$

In the equilibrium state $\delta E_{\text {in }} \simeq \delta E_{\text {diss }}$, hence

$\frac{n}{N^{2}} \simeq \frac{4\left\langle\delta B^{2}\right\rangle}{j_{\max }^{2}} \simeq \frac{2}{t_{\mathrm{S}}}$.

Assuming that these currents are uniformly distributed over the grid, the characteristic distance between them is

$l \simeq \frac{N}{\sqrt{n}}=\frac{1}{2} \frac{j_{\max }}{\sqrt{\left\langle\delta B^{2}\right\rangle}}$.

\subsection{Grid size effects}

To investigate the grid size effects, we take grids consisting of $30 \times 30-400 \times 400$ cells, the conditions of anomalous resistivity dissipation, and $j_{\max }=5$. In this case we obtain the distributions that are very close to Gaussian for the largest grid. All the simulations were performed during $10^{6}$ time steps. The results are shown in Fig. 4 for different sizes of the grid. For the $30 \times 30$ grid, the distribution is nicely fit by a power law of index -3.1 for almost 3 energy decades. As the grid size is increased, the bulk of the distribution becomes closer to a Gaussian one, while the high-energy tail retains a power-law shape. For the $50 \times 50$ grid the power law shape of the tail is observed only for one energy decade, the estimate of the index being -2.9 . For the $64 \times 64$ grid the power law tail is again shorter and the index is approximately equal to -4.3 . For the grid $100 \times 100$ the distribution is practically indistinguishable from Gaussian over the whole energy range. 

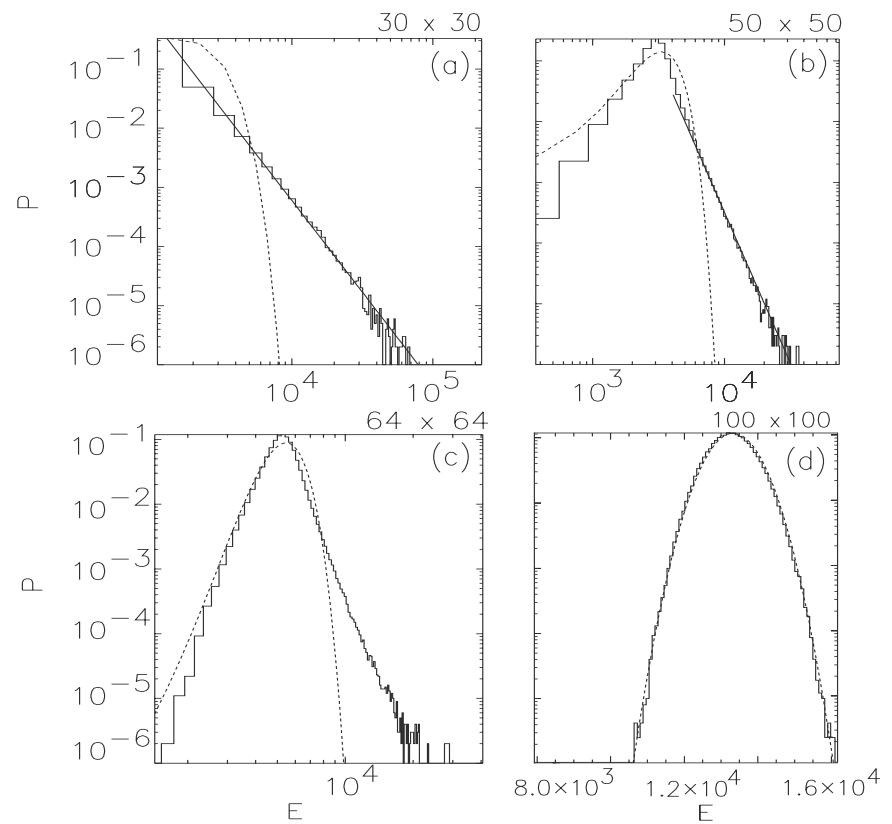

Fig. 4. Probability distribution function of the dissipated energy versus energy released (solid lines). Dotted lines represent the best fits by Gaussian distributions. The results are obtained for unipolar random source and anomalous resistivity dissipation with a threshold $j_{\max }=5$. The grid sizes are the following: a) $30 \times 30$; b) $50 \times 50$; ) $64 \times 64$; d) $100 \times 100$.

Eventually, for a large grid size the whole distribution becomes Gaussian. This means that the character of spatial correlations is changed. For small grids, spatial correlations decay rather slowly so that they extend over the whole grid. With the growth of the grid size, the exponential tails of the spatial correlations appear yielding the correlation length smaller than the grid size. The fact that the power-law distributions transform into Gaussian with the increase in the size of the system considered has already been observed in some real sandpile experiments (Held et al. 1990) and in some forest-fire models (Grassberger 1991).

Now we proceed to the discussion of simulation results for various unipolar and dipolar sources and for both dissipation criteria using sufficiently large grids to avoid unphysical effects.

\subsection{Random unipolar sources}

Simulations on a $200 \times 200$ grid were performed for $2 \times 10^{5}$ time steps and both types of dissipation (anomalous resistivity and reconnection). The distributions of dissipated energy due to anomalous resistivity with the thresholds $j_{\max }=5,30,100$ are shown in Fig. 5. Figure 6 represents the same PDF but for dissipation provided by reconnection.

The reason for the PDF shown in Fig. 5c to have multiple extrema is easy to understand. Since the dissipated energy in each event is of the form $\left(j_{\max }+\varepsilon\right)^{2}$, where $0<\varepsilon<2$, for large $j_{\max }$, the peaks separated by $j_{\max }^{2}$
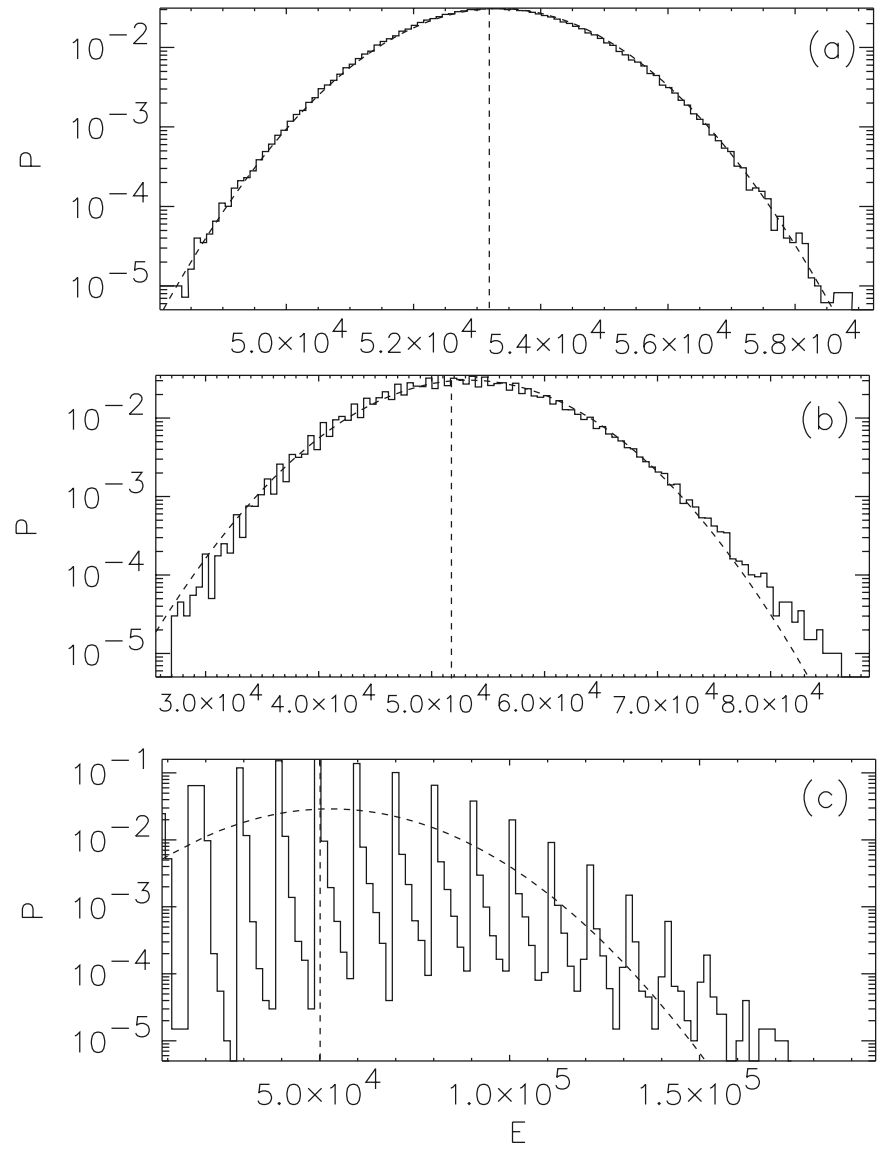

Fig. 5. Probability distribution function of the dissipated energy versus energy released (solid lines). Dotted lines represent the best fits by Gaussian distributions. The results are obtained for unipolar random source and anomalous resistivity dissipation. The threshold currents $j_{\max }$ are the following: a) $j_{\max }=5$; b) $j_{\max }=30$; c) $j_{\max }=100$.

appear. Assuming that $\varepsilon$ is a uniformly distributed random variable, we can get that the width of the peak number $K$ is of the order of $K\left\langle\varepsilon^{2}\right\rangle$. Hence, the discrete character of the PDF appears for the smallest values of the dissipated energy and large $j_{\max }$ (see Figs. 5b-c).

From Figs. 5 and 6, one can see that the smaller current threshold (as compared to the source amplitude) results in a PDF of dissipated energy that is close to normal. With the growth of $j_{\max }$, a high energy nonthermal tail appears. Although the average value of the dissipated energy does not depend upon $j_{\max }$, the deviations from Gaussian distribution in the tail increase with $j_{\max }$ and are more pronounced for dissipation due to reconnection. For the dissipation due to reconnection, all three distributions have non-Gaussian tails that can be approximated by power-law. With the increase of $j_{\max }$ the tails become more extended and the index of the power-law distribution decreases. This signifies that the stronger deviations from a Gaussian distribution appear for larger values of the threshold.

The formation of such a tail is not related to the increase of the correlation length, because the largest 

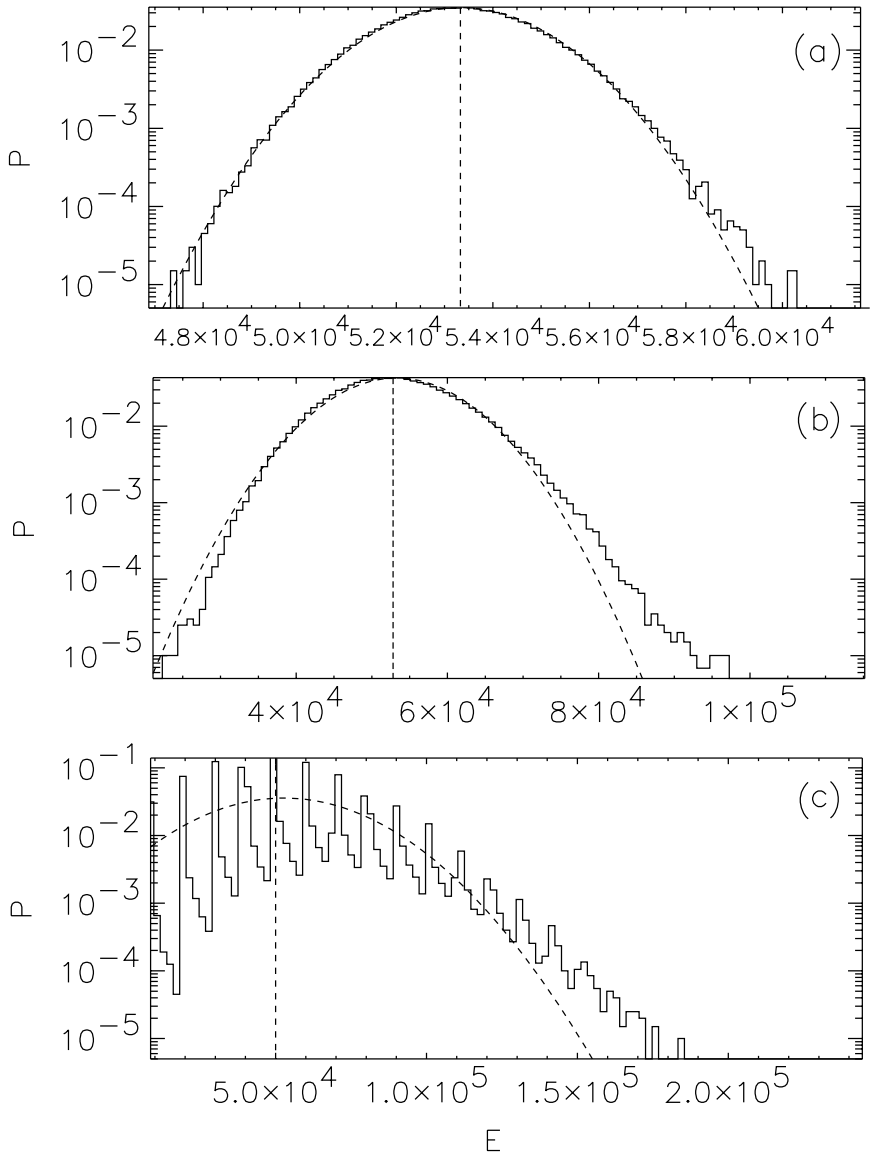

Fig. 6. Same as for Fig. 5, but for the reconnection-like dissipation.

magnetic field correlation length is about 20 for moderate threshold, $j_{\max }=5$, in the case of reconnection-like dissipation. Let us also notice that the average correlation length is smaller when anomalous resistivity dissipation is considered and decreases as $j_{\max }$ increases (Podladchikova et al. 1999).

The average number of dissipated currents decreases with the increase of the threshold. Indeed, it easily seen from Eq. (3) that for $j_{\max }=1$ we have

$\frac{n}{N^{2}}=\frac{4}{3}$,

i.e., each current is dissipated at each time step and dissipative events occur almost everywhere on the grid thereby creating long-range correlations. For large $j_{\max }$, the ratio $n / N^{2}$ becomes small, e.g., for $j_{\max }=100$ we have $n / N^{2} \simeq 3 \times 10^{-4}$ that corresponds to the uncorrelated dissipative events with relatively small overlapping.

Thus, for large $j_{\max }$ the dissipation is much faster than the action of the magnetic field source, i.e., the conditions of time scale separation are better satisfied.

\subsection{Ulam map source}

Despite the deterministic nature of the magnetic field increment $\delta B$, without dissipation the magnetic field in each
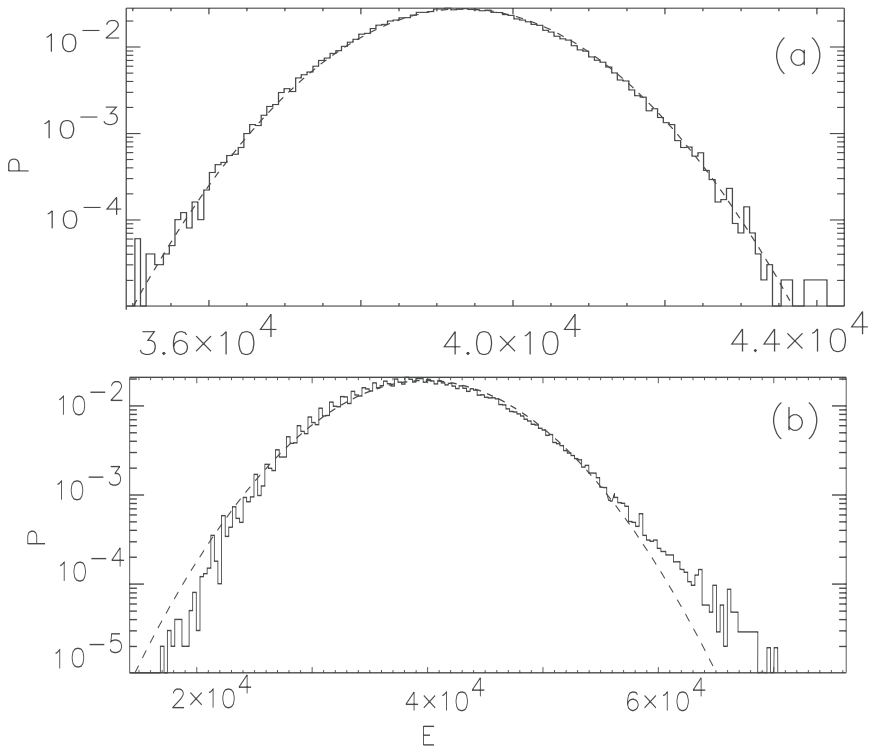

Fig. 7. Probability distribution function of the dissipated energy versus energy released (solid lines). Dotted lines represent the best fits by Gaussian distributions. The results are obtained for Ulam map source and $j_{\max }=30$. The dissipation laws are the following: a) anomalous resistivity dissipation; b) reconnection-type dissipation.

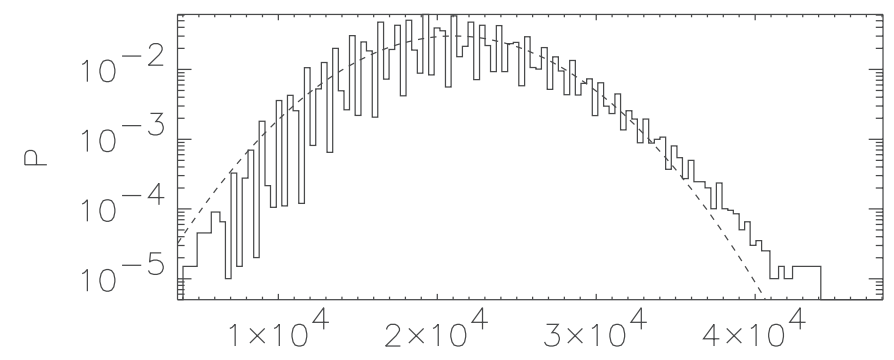

Fig. 8. Probability distribution function of the dissipated energy versus energy released (solid lines). Dotted lines represent the best fits by Gaussian distributions. The results are obtained for the Geisel map source and anomalous resistivity dissipation. Threshold current $j_{\max }=30$.

cell exhibits a Brownian motion with Gaussian statistics after a large number of steps. Indeed, each $\delta B$ has the same PDF with a finite variance. Thus, the dependence of the PDF upon the critical current and the dissipation mechanism have manifested itself in similar tendencies as random sources do. The PDFs for moderate critical current, $j_{\max }=5$, are close to Gaussian, for $j_{\max }=30$ the small deviations from Gaussian distribution begin to appear for high energies, and for $j_{\max }=100$ there is clear evidence of the presence of the high energy suprathermal tail. The tail is much better pronounced for the dissipation due to reconnection (Fig. 7).

\subsection{Geisel map lattice}

Another source of the magnetic field is provided by the Geisel map. It was used as a source of the field rather than the increment of the field. As it was discussed above, 

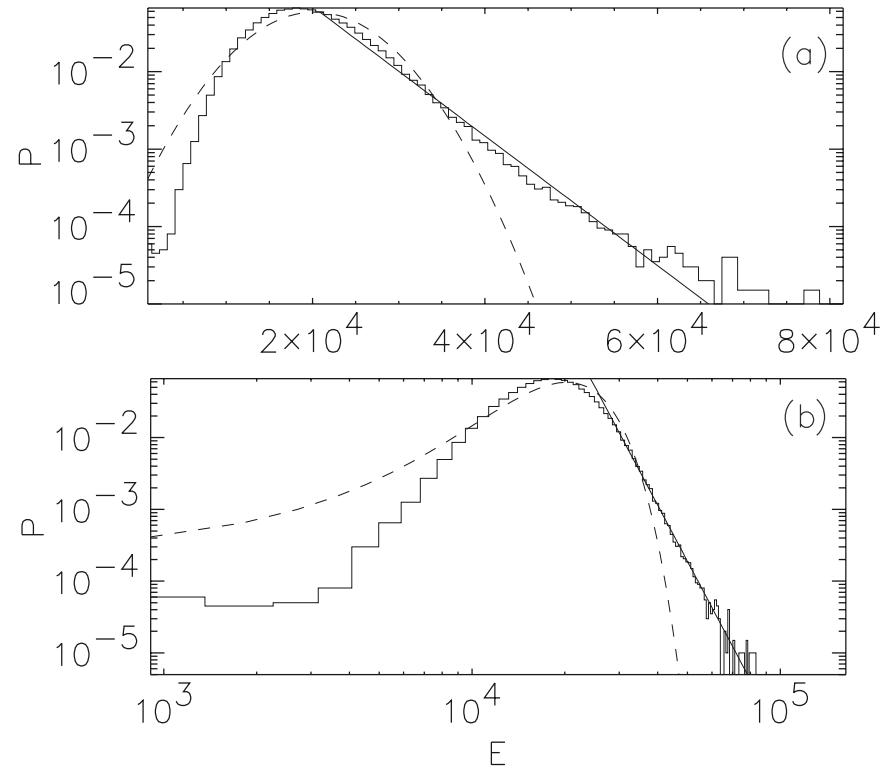

Fig. 9. Probability distribution function of the dissipated energy versus energy released (solid lines). Dotted lines represent the best fits by Gaussian distributions. The results are obtained for Geisel map source and dissipation due to reconnection. Threshold current is $j_{\max }=30$.

this map exhibits the time evolution in each cell quite similar to anomalous (non-Brownian) diffusion. The growth of the magnetic field variance is slower than for Brownian motion. Such a behavior of the walker would be called subdiffusive. As a result, the variance is smaller than for the random source. This causes some differences observed in the behavior of the PDF of the dissipated energy. If the dissipation is provided by anomalous resistivity, the PDFs obtained with this map and the random source are quite similar except for the width of the distribution. Figure 8 represents the PDF obtained for the Geisel map and $j_{\max }=30$.

The smaller current threshold (as compared to the source amplitude) results in a PDF of dissipated energy that is close to normal. With the growth of $j_{\max }$, a high energy non-thermal tail appears. The deviations from Gaussian distribution in the tail increase with $j_{\max }$. With the increase of $j_{\max }$ the tails become more extended.

In this case the range of energies is slightly less than for the Ulam map, the distribution being quite jagged, all the other features of the distributions seem to be similar.

Figure 9 shows the PDF obtained for the same Geisel map source, dissipation due to reconnection, and $j_{\max }=$ 30. A pronounced tail of the distribution is evidenced, thereby confirming the tendencies already observed for other types of sources. A difficulty that one encounters when analyzing such PDFs is illustrated in Fig. 9. In Fig. 9a, the PDF is represented in semi-logarithmic scale and the distribution seems to have an exponential tail. In Fig. 9b the same distribution is shown in the log-log representation, where the same tail can be treated as a powerlaw-type distribution with the index -3.1 . This problem

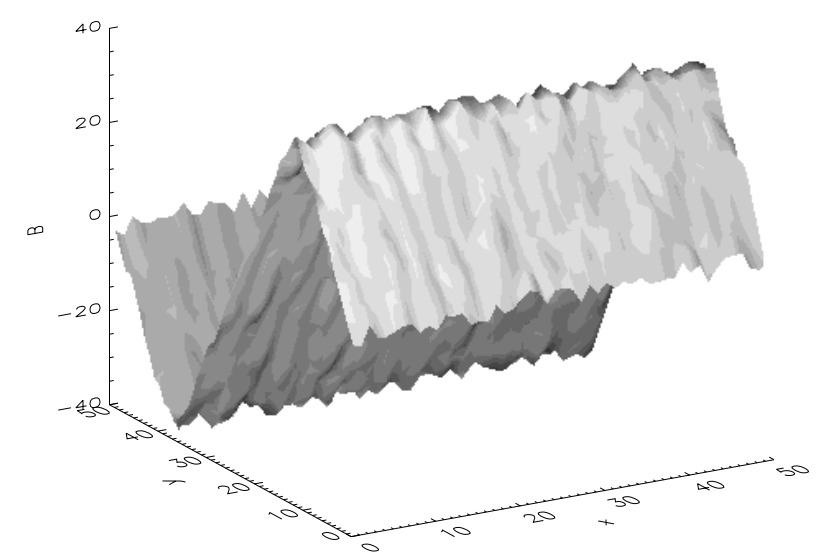

Fig. 10. Magnetic field structure observed in the simulations with the dipolar random source, anomalous resistivity dissipation, and a threshold current $j_{\max }=5$.

is related to the fact that the tail extends over a rather short range of about one energy decade. This problem will be treated in more detail elsewhere (Podladchikova et al. 2001).

\subsection{Dipolar source}

When the effects due to the bi-polarity are modeled, the grid is split into two equal parts. For one half of the grid, the value of the magnetic field increment is chosen randomly from the set $\{-1.5,-0.5,0.5\}$, for another half the set $\{1.5,0.5,-0.5\}$ is used. The global inhomogeneity of the dipolar source results in the formation of a strongly localized current layer in the transition region between the two parts of the system. The grid size is $100 \times 100$.

The dissipation provided by reconnection is not always able to stabilize the field growth. However, for dissipation due to anomalous resistivity a stationary state always exists. Further we consider only the case with anomalous resistivity dissipation.

The energy of the dipolar structure grows faster than in the unipolar case, and a stationary state is quickly reached. The dipolar structure of the magnetic field is presented in Fig. 10. This structure is stationary. Therefore the spatial correlations of magnetic field are long and quasi-stationary.

Such a system differs from the unipolar one with the random source. We observe, as could be expected, that the dissipation events are more intense within the transition region where the magnetic field changes its sign. In addition, in the dipolar case the position of the PDF peak value on the energy axis is significantly shifted towards high energies with the increase of the threshold. Such a property can be explained by the presence of the localized current layer in the vicinity of the neutral sheet. The characteristic dependence of the PDFs upon the dissipated energy in dipolar case displays supra-thermal high energy tails which were not observed in the unipolar case with anomalous resistivity (see Fig. 11). The characteristic indices for $j_{\max }=50,100$, and 230 are approximately equal 

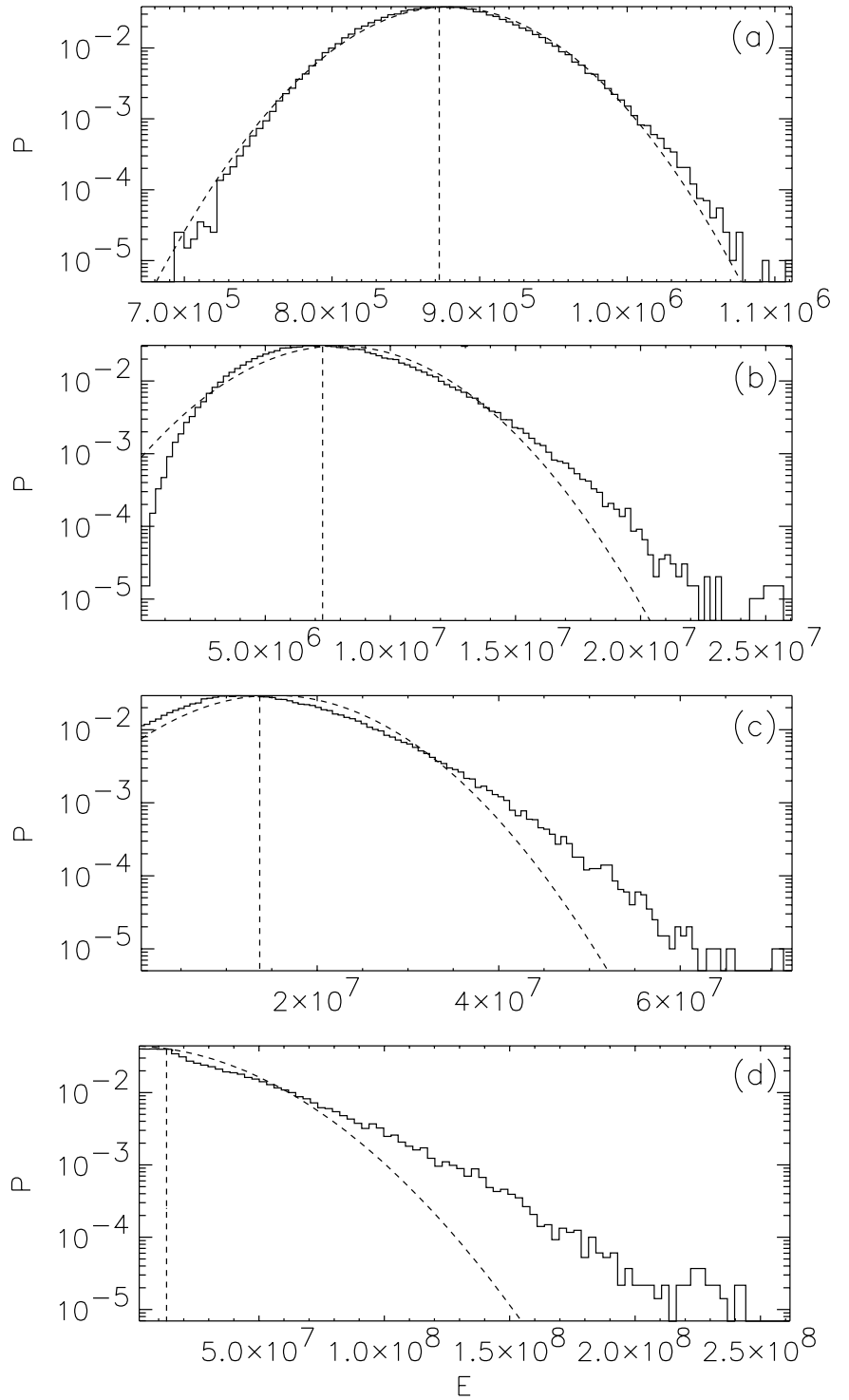

Fig. 11. Probability distribution function of the dissipated energy versus the energy released (solid lines). Dotted lines represent the best fits by Gaussian distributions. The results are obtained for dipolar random source and anomalous resistivity dissipation. The duration of calculations was $2 \times 10^{5}$ time steps. The threshold currents $j_{\max }$ are the following: a) $j_{\max }=5$; b) $j_{\max }=50$; c) $j_{\max }=100$; d) $j_{\max }=230$.

to $-1.7,-1.9$, and -2.3 , respectively. For $j_{\max }=230$, the time series of the dissipated energy is shown in Fig. 12.

\section{Discussion and conclusions}

To study coronal heating due to dissipation of small-scale current layers, we have performed a statistical analysis of a simple cellular automata model. Its principal difference to previous ones is that the system is driven by small-scale homogeneously distributed sources acting on the entire grid for each time step. The idea to consider small-scale sources is similar to the one proposed by Benz \& Krucker, i.e. that the heating occurs on the level of the chromosphere, thus the magnetic field structures, the dissipation

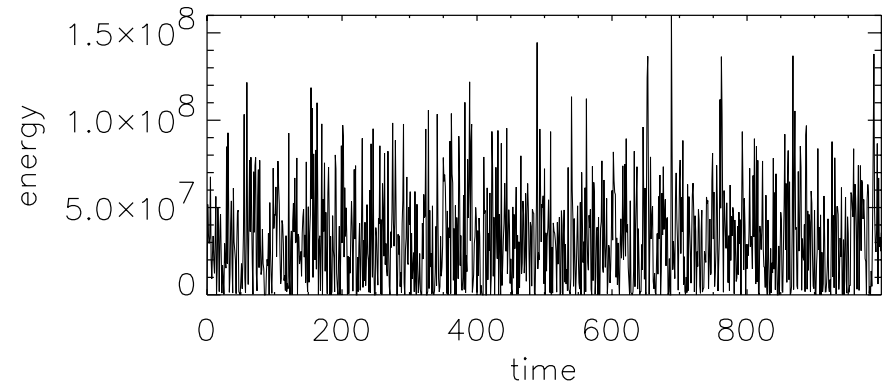

Fig. 12. Fragment of time series of the dissipated energy, for $j_{\max }=230$, dipolar random source, anomalous resistivity dissipation.

of which supplies the energy for the heating, are also of a small scale.

The magnetic field sources we use are either of the following types:

- random source;

- deterministic chaotic map (Ulam map), both for the magnetic field increment;

- Geisel map (coupled map lattice) that describes the time evolution of the magnetic field in each cell similar to anomalous (non-Brownian) diffusion.

We consider two mechanisms of small-scale dissipation to occur:

- the first is used to model anomalous resistivity dissipation:

- the second is used to model the local reconnection.

The first one is supposed to be similar to Joule dissipation but in collisionless plasma. It relies on the criterion that any local current whose magnitude exceeds a predetermined threshold value must dissipate.

In the second case we assume that the dissipation occurs when the mentioned above condition is satisfied (this does not mean that the threshold in the real physical system should be the same in the two cases) and, in addition, the magnetic fields vectors in neighboring cells should have opposite signs. This mimics the presence of the null point of the magnetic field in the reconnectiontype configurations.

The characteristic under investigation is the total energy of all simultaneously dissipated currents. Our observations can be summarized as follows.

Small-scale magnetic field sources and localized energy dissipation mechanisms can result in large-scale correlations of the magnetic field. In our calculations, the energy sources dominate in larger scales while the dissipation in the smaller ones. Thus, the system behaves as having the energy cascade from large scales to smaller ones. However, during the evolution of the system, large-scale correlations are formed with a characteristic length significantly larger than that of the source and dissipation.

For all three types of the magnetic field sources considered, the obtained PDF of the dissipated energy is close 
to Gaussian distribution when relatively small threshold currents, $j_{\max }<10$, are chosen. The dependence of the PDF of the dissipated energy upon statistical properties of the source for all three types of the sources considered is rather weak and requires an additional thorough analysis to be performed.

In the case of reconnection-type dissipation, the deviations from Gaussian distribution are stronger than for anomalous resistivity dissipation. For large values of the threshold current density, we observe a high-energy suprathermal tail which has a shape similar to a powerlaw distribution. The time-averaged spatial correlations are exponentially decaying. In this sense our model can not be considered as a self-organized critical system. However, sometimes long-range (power-law type) correlations are observed.

To model a dipolar global magnetic field structure, we used an inhomogeneous distribution of the magnetic field source. It takes a small average positive value in one part of the grid and a negative value in the other, such that the average over the whole grid is zero. The system does not reach any stationary state in the case of the reconnection type dissipation. In the case of anomalous resitivity it saturates and the stationary state is established. In this last case, the deviations from the Gaussian become stronger with the increase of the threshold. This effect is associated with the decrease of the thickness of the current layer where the dissipation is concentrated.

The statistical analysis of the frequency distribution of such heating events always shows a larger absolute value for the exponent for the more energetic reconnectionproduced events than for the anomalous resistivity heating events. This tendency seems to be similar to that found by Benz \& Krucker (1998) who studied the emission measure increases. They pointed out that the statistical properties of the faint events that occur in the intra-cell regions of the quiet Sun manifest quite small deviations from Gaussian distributions, while supposed nanoflares that are associated with the network boundaries have more prominent enhancements and stronger deviations. Taking this into account we come to the conclusion that the quantitative difference between faint and strong heating events reported in (Krucker \& Benz 2000) can probably be explained by two different mechanisms of magnetic field dissipation that we used in our model.

However, our current work represents only the first step in the development of a model. In particular, several elements may be better adapted to real physical processes. Further effects should be included for direct comparison with the observations, such as more a detailed description of the respective time scales of the instabilities or particle acceleration, thermal/non-thermal processes or outgoing flows escaping from the reconnection region. Also, it can be reasonably assumed that in the case of the anomalous resistivity the dissipation of the current can be only partial, and that the two dissipation mechanisms can coexist. The energy re-distribution between different particle species, as noted in the Appendices, can also be taken more carefully into account. Thus, at this stage we can only conclude that some tendencies of our model seem to be in a good qualitative agreement with the experimental ones.

Acknowledgements. The authors would like to acknowledge Professor A. Benz for fruitful comments that helped to improve this paper. The authors are thankful to V. Lobzin, T. Dudok de Wit, S. Koutchmy and S. M. Levitsky for fruitful and useful discussions. B. Lefebvre is grateful to JSPS for financial support. O. Podladchikova is grateful to French Embassy in Ukraine for the financial support.

\section{Appendix A: Anomalous resistivity}

In this appendix, we explain in more detail the physics of the "anomalous resistivity" mentioned in our paper. The idea is based on the possibility of the exchange of the pulse and energy between the electrons and the ions due to the instability development and the appearance of the turbulent state in the plasma. The "conventional" expression for the conductivity reads

$\sigma=\left(n e^{2}\right) /\left(m_{\mathrm{e}} \nu\right)$,

where $n$ is the plasma density, e, $m_{\mathrm{e}}$-electron charge and mass respectively, $\nu$ is the collision frequency of the electrons with ions. In collisionless plasma the collision frequency is negligible and the collisional conductivity is infinite. However the electrons (that carry the major part of the electric current) can excite collective oscillations of the electrons as well as ions, and transfer part of their impulse and energy to these oscillations. This results in an anomalous loss of the electron pulse and energy, and, consequently, to the decrease of their directed velocity, i.e. the decrease of the current. Such a process can be characterized by an "effective collision frequency" $\nu_{\text {eff }}$. To calculate this characteristic frequency one can consider the pulse conservation law in the system that consists of electrons and waves. To this end one should evaluate this effect as the action of the friction force that decelerates the electron flow. The pulse loss per unit time can be written as follows:

$\nu_{\mathrm{eff}} m_{\mathrm{e}} n \boldsymbol{U}_{\mathrm{d}}=-\boldsymbol{F}_{\mathrm{fr}}$

where $U_{\mathrm{d}}$ is the directed velocity of the electron flow that carries the current, and $F_{\text {fr }}$ the friction force that acts on the electrons. The same decrease of the electron pulse can be estimated as the increase of the pulse of the waves emitted by the electrons taking into account that the change of the pulse of the waves is described by the following expression:

$\frac{\mathrm{d} P_{\mathrm{w}}}{\mathrm{d} t}=2 \int \gamma_{k} W_{k} \frac{\boldsymbol{k}}{\omega_{k}} \frac{\mathrm{d}^{3} k}{(2 \pi)^{3}}$

where $\gamma_{k}$ is the linear increment of the instability and $W_{k}$ is the spectral energy density of waves. The assumption that the pulse of the system consisting of electrons and waves is conserved results in the equality of these two 
expressions, thus the effective collision frequency can be defined as:

$\nu_{\mathrm{eff}}=\frac{2}{m_{\mathrm{e}} n U_{\mathrm{d}}^{2}} \int \gamma_{k} W_{k} \frac{\left(\boldsymbol{U}_{\mathrm{d}} \boldsymbol{k}\right)}{\omega_{k}} \frac{\mathrm{d}^{3} k}{(2 \pi)^{3}}$.

Thus the estimate of the level of the wave turbulence taking into account the nonlinear saturation mechanism allows us to solve the problem.

When the current flows in the direction perpendicular to the magnetic field, the two major types of instability that give rise to the anomalous resistivity are modified Buneman instability and the instability with respect to the generation of the so-called electron-acoustic modes. The final effect of the anomalous turbulent resistivity is the transfer of the energy from the electrons to ions, because the damping of these waves takes place mainly due to their interaction with ions.

An important physical characteristics of the anomalous resistivity phenomenon is the ratio of the energy dissipated by ions and to the energy dissipated by the electrons. If the characteristic drift velocity of the electrons with respect to ions is $\boldsymbol{V}_{\mathrm{d}}$, and we have the knowledge of the spectral characteristics of the wave spectrum excited, let us assume for instance that the characteristic frequency of the waves excited due to the instability is $\omega_{\boldsymbol{k}}$, and that characteristic wave vector of the unstable waves is $\boldsymbol{k}$, then this ratio can be estimated by:

$\frac{\mathrm{d} E_{\mathrm{e}}}{\mathrm{d} t} / \frac{\mathrm{d} E_{\mathrm{i}}}{\mathrm{d} t} \sim \frac{\int \gamma_{\boldsymbol{k}} W_{\boldsymbol{k}} \frac{\left(\boldsymbol{k} \boldsymbol{V}_{\mathrm{d}}\right)}{\omega_{k}} \mathrm{~d}^{3} \boldsymbol{k}}{\int \gamma_{\boldsymbol{k}} W_{\boldsymbol{k}} \mathrm{d}^{3} \boldsymbol{k}} \sim \frac{\left(\boldsymbol{k} \boldsymbol{V}_{\mathrm{d}}\right)}{\omega_{\boldsymbol{k}}}$

for typical instabilities, where this ratio is approximately 1 .

So, the final result of the anomalous resistivity is quite similar to the Joule heating of the ion component of the plasma. The energy dissipation that heats the plasma can be represented as:

$Q=j^{2} / \sigma_{\text {eff }}$.

This phenomenon was observed experimentally in the laboratory plasma (Eselevich et al. 1971).

\section{Appendix B: Reconnection}

There are several differences between the reconnection process and anomalous resistivity. One of them is related to the change of the magnetic field topology in the first case while in the second there are only quantitative variations of the basic characteristics of the magnetic field configuration.

Another difference is that while the diffusion processes in the first case provide heating (comparable for ions and electrons, see appendix), reconnection converts magnetic energy mostly into particle (mainly ion) acceleration. Then energetic beams may provide heat, but this is only an indirect consequence of the reconnection. Another difference, quite important for observations, is a difference in time scales. The reconnection is supposed to be a rapid energy release while the anomalous resistivity is relatively slow diffusive process.

Moreover, as it was shown analytically and in computer simulations, the reconnection process can under certain conditions look like an explosive event. During the reconstruction of the magnetic field topology, the component of the magnetic field perpendicular to the background magnetic field and to the direction of the current can grow explosively. The spatio-temporal dynamics of the magnetic field can be described by the following expression:

$\boldsymbol{B}=B_{0 x} \tanh \left(\frac{z}{L}\right) \boldsymbol{e}_{x}+B_{z}(t) \sin (k x) \boldsymbol{e}_{z}$,

where

$B_{z}(t)=\frac{B_{0 z}}{1-t / \tau_{\operatorname{expl}}}$.

(1984). Here $B_{0 x}$ is the magnitude of the background surrounding magnetic field that is supplied by the current carried along the $y$ axes, and $\tau_{\text {expl }}$ is the characteristic time of the magnetic and electric fields variations. $B_{0 z}$ is the initial amplitude of the perturbation of the normal component of the magnetic field. $B_{z}$ grows to infinity in a finite time, although this formal solution is valid only when the amplitude of this perturbation is smaller than the background field $B_{0} x$ and then saturates. This explosive growth of nonlinear perturbations results in the similar increase of the inductive electric field. This process results in the rapid acceleration of electrons and ions by the inductive electric field in the region where the particles are unmagnetized.

Another observational feature of the reconnection process consists of the presence of the macroscopic fluxes around the reconnection site, with the characteristic velocities of the order of the Alfvén speed in the vicinity of the reconnection site. These flows can give rise to the replenishing of the lower density regions by the material from the reconnection site if the density there is larger or even comparable with the density of the surrounding plasma. A part of the energy released is supposed to be transformed into the kinetic energy of accelerated charged particles. It is known that the energy transferred to particles grows with the increase of their mass as $M^{1 / 3}$, the heaviest ones being the most effectively accelerated (Vekstein \& Priest 1995). In turn, these particles can generate electromagnetic radiation providing experimental signatures of the heating events.

\section{References}

Abramenko, V. I., Yurchyshyn, V. B., \& Carbone, V. 1999, in Magnetic fields and Solar Processes, ESA SP-448, 2, 679

Aschwanden, M. J., Tarbell, T. D., Nightingale, R. W., et al. 2000, ApJ, 535, 1047

Bak, P., Tang, Ch., \& Wiesenfeld, K. 1988, Phys. Rev. A, 36, 364

Benz, A. O., Krucker, S., Acton, L. W., et al. 1997, A\&A, 320, 993

Benz, A. O., \& Krucker, S. 1998, Sol. Phys., 182, 349 
Benz, A. O., \& Krucker, S. 1999, A\&A, 341, 286

Berghmans, D., Clette, F., \& Moses, D. 1998, A\&A, 336, 1039

Biskamp, D. 1993, Nonlinear Magnetohydrodynamics (Cambridge University Press)

Boffetta, G., Carbone, V., Giuliani, P., et al. 1999, Phys. Rev. Lett., 83, 4662

Crosby, N. B., Aschwandnen, M. J., \& Dennis, B. R. 1993, Sol. Phys., 143, 275

Einaudi, G., \& Velli, M. 1994a, Space Sci. Rev., 68, 97

Einaudi, G., \& Velli, M. 1994b, in Advances in Solar Physics, Lecture Notes in Physics, ed. G. Belvedere, M. Rodono, \& G. M. Simnett (Springer, Berlin), 432, 149

Einaudi, G., \& Velli, M. 1999, Plasma Phys., 6, 4146

Einaudi, G., Velli, M., Politano, H., et al. 1996, ApJ, 457, L113

Eselevich, V. G., Eskov, A. G., Kurtmulaev, R. H., et al. 1971, Zh. Eksp. Teor. Fiz, 60, 2079

Frisch, U. 1995, Turbulence (Cambridge University Press)

Galeev, A. A., \& Sagdeev, R. Z. 1979, in Rev. Plasma Phys., ed. M. A. Leontovich, 7, 3

Galeev, A. A., \& Sagdeev, R. Z. 1984, in Basics of Plasma Physics, ed. A. A. Galleev, \& R. N. Sudan, vol. 2, 271

Galeev, A. A. 1984, in Basics of Plasma Physics, ed. A. A. Galleev, \& R. N. Sudan, vol. 2, 305

Geisel, T., \& Thomae, S. 1984, Phys. Rev. Lett., 52, 1936

Georgoulis, M. K., \& Vlahos, L. 1996, ApJ, 469, L135

Georgoulis, M. K., Velli, M., \& Einaudi, G. 1998, ApJ, 497, 957

Georgoulis, M. K., \& Vlahos, L. 1998, A\&A, 336, 721

Georgoulis, M. K., Kluiving, R., \& Vlahos, L. 1995, Phys. A, 218,191

Georgoulis, M. K., Vilmer, N., \& Crosby, N. B. 2001, A\&A, 367,326

Grassberger, P., \& Kantz, H. 1991, J. Stat. Phys., 63, 685

Harrison, R. A. 1997, in The Corona and Solar Wind Near Minimum Activity, ESA SP-404, 7

Held, G. A., Solina, D. H., Solina, H., et al. 1990, Phys. Rev. Lett., 65, 9, 1120

Jensen, H. J. 1998, Self-Organized Criticality (Cambridge University Press)

Isliker, H., Anastasiadis, A., Vassiliadis, D., et al. 1998, A\&A, 335,1085
Kaneko, K. (ed.) 1992, Chaos: Focus issue on coupled map lattices, Chaos 2(3)

Koutchmy, S., Hara, H., Suematsu, Y., et al. 1997, A\&A, 320, L33

Krucker, S., \& Benz, A. O. 1998, ApJ, 501, L213

Krucker, S., \& Benz, A. O. 2000, Sol. Phys., 191, 341

Lin, R. P., Schwartz, R. A., Kane, S. R., et al. 1984, ApJ, 283, 421

Lu, E. T., \& Hamilton, R. J. 1991, ApJ, 380, L89

Lu, E. T., Hamilton, R. J., McTiernan, J. M., et al. 1993, ApJ, 412,841

Mitra Kraev, U., \& Benz, A. O. 2001, A\&A, in press

Parker, E. N. 1988, ApJ, 330, 474

Parnell, C. E., \& Jupp, P. E. 2000, ApJ, 529, 554

Petchek, H. E. 1964, in AAS/NASA, Symp. Physics of Solar Flares, ed. W. N. Hess (NASA, Washington, DC), 425

Podladchikova, O., Krasnoselskikh, V., \& Lefebvre, B. 1999, in Magnetic fields and Solar Processes, ESA SP-448, 1, 553

Podladchikova, O., \& Krasnoselskikh, V. 2001, in preparation

Priest, E. R., Foley, C. R., Heyvaerts, J., et al. 1998, Nature, 393,545

Priest, E. R., Foley, C. R., Heyvaerts, J., et al. 2000, ApJ, 539, 1002

Priest, E. R., \& Forbes, T. 2000, Magnetic Reconnection (Cambridge University Press)

Shimizu, T., Tsuneta, A., Acton, L., et al. 1994, ApJ, 422, 906

Sornette, D., Johansen, A., \& Dornic, I. 1995, J. Phys. I France, 5,325

Somov, B. V., \& Syrovatsky, S. I. 1977, Sol. Phys., 35(2), 393

Syrovatsky, S. I. 1981, ARA\&A, 19, 161

Syrovatsky, S. I. 1982, Sol. Phys., 76(1), 3

Vassiliadis, D., Anastasiadis, A., Georgoulis, M., et al. 1998, ApJ, 509, 53

Vekstein, G. E., \& Priest E. R. 1995, Phys. Plasmas, 2(8), 3169

Vespigniani, A., \& Zapperi, S. 1998, Phys. Rev. E, 57(6), 6345

Vlahos, L. 1994, Space Sci. Rev., 68, 39

Vlahos, L., Georgoulis, M., Kluiving, R., et al. 1995, A\&A, 299, 897

Woo, R., \& Habbal, S. R. 1997, ApJ, 474, L139 